\title{
HOMOTOPY CLASSES IN SOBOLEV SPACES AND ENERGY MINIMIZING MAPS
}

\author{
BY BRIAN WHITE
}

Let $M$ and $N$ be compact Riemannian manifolds. The energy of a lipschitz $\operatorname{map} f: M \rightarrow N$ is $\int_{M}|D f|^{2}$ (where $|D f(x)|^{2}=\sum\left|\partial f / \partial x_{i}\right|^{2}$ if $x_{1}, \ldots, x_{m}$ are normal coordinates for $M$ at $x$ ). Mappings for which the first variation of energy vanishes are called harmonic. The identity map from $M$ to $M$ is always harmonic, but it may be homotopic to mappings of less energy. For instance, the identity map on $S^{3}$ is homotopic to mappings of arbitrarily small energy (namely, conformal maps that pull points from the North Pole toward the South Pole). That suggests the question: For which manifolds $M$ is the identity map homotopic to maps of arbitrarily small energy? In this paper we give the simple answer: Those $M$ such that $\pi_{1}(M)$ and $\pi_{2}(M)$ are both trivial. More generally, we consider energy functionals like $\Phi(f)=\int_{M}|D f|^{p}$ and ask:

(1) When is the infimum of $\Phi(f)$ in some homotopy class of mappings $f: M \rightarrow N$ nonzero?

(2) When is the infimum of $\Phi(g)$ (among maps satisfying some homotopy condition) actually attained?

To answer such questions, it is convenient to regard $N$ as isometrically embedded in a euclidean space $\mathbf{R}^{\nu}$ and to work with the Sobolev norm

$$
\|f\|_{1, p}=\left(\int_{M}|f|^{p}\right)^{1 / p}+\left(\int_{M}|D f|^{p}\right)^{1 / p}
$$

(where $f: M \rightarrow \mathbf{R}^{\nu}$ has distribution derivative $D f$ ) and with the associated Sobolev spaces,

$$
L^{1, p}(M, N)=\left\{f: M \rightarrow \mathbf{R}^{\nu} \mid f(x) \in N \text { for a.e. } x, \text { and }\|f\|_{1, p}<\infty\right\}
$$

and

$$
W^{1, p}(M, N)=\text { the closure of }\{\text { lipschitz maps } f: M \rightarrow N\} \text { in } L^{1, p}(M, N) .
$$

Say that two continuous maps $f, g: M \rightarrow N$ are $k$-homotopic (or have the same $k$-homotopy type) if their restrictions to the $k$-dimensional skeleton of some triangulation of $M$ are homotopic. We have the following theorem about $W^{1, p}(M, N)$ (where $[p]$ is the integer part of $p$ ).

THEOREM 1. Two lipschitz maps are in the same connected component of $W^{1, p}(M, N)$ if and only if they are $[p]$-homotopic. Consequently every map in $W^{1, p}(M, N)$ has a well-defined $[p]$-homotopy type. Furthermore, the set of

Received by the editors January 24, 1985.

1980 Mathematics Subject Classification (1985 Revision). Primary 58E20, 55P10; Secondary $46 \mathrm{E} 35$. 
lipschitz maps homotopic to a given map $f$ is dense (with respect to $\|\cdot\|_{1, p}$ ) in the connected component containing $f$.

As a corollary we have the answer to (1).

COROLlaRY. The infimum of $\Phi(g)$ among lipschitz maps $g: M \rightarrow N$ homotopic to a given lipschitz map $f: M \rightarrow N$ is equal to the infimum of $\Phi(g)$ among all lipschitz maps that are merely $[p]$-homotopic to $f$. In particular, the infimum is 0 if and only if the restriction of $f$ to the $[p]$-skeleton of $M$ is homotopically trivial.

The space $W^{1, p}(M, N)$ is not, however, suitable for studying existence questions such as (2) because it lacks nice compactness properties. In $L^{1, p}(M, N)$, on the other hand, closed bounded sets are compact in the weak topology. We have

THEOREM 2. Every $f \in L^{1, p}(M, N)$ has a well-defined [p-1]-homotopy type. If $f_{i} \in L^{1, p}(M, N)$ is a $\|\cdot\|_{1, p}$-bounded sequence of functions with a given $[p-1]$-homotopy type, and if $f_{i}$ converges weakly to $f$, then $f$ has the same $[p-1]$-homotopy type.

This gives the answer to (2).

COROLlaRY. The infimum of $\Phi(g)$ among all maps $g \in L^{1, p}(M, N)$ with a given $[p-1]$-homotopy type is attained.

In case $p=2$, then $\Phi(g)$ is the ordinary energy of $g$, and the minimizing map $g$ is locally energy minimizing in the sense studied by Schoen and Uhlenbeck $[\mathbf{S U 1}, 2]$. By combining the above existence result with their regularity theorems, we obtain

THEOREM 3. In every 1-homotopy class of mappings in $L^{1,2}(M, N)$, there is a map $g$ of least energy. Such a map is a smooth harmonic map except on a closed set $K \subset M$ of Hausdorff dimension $\leq \operatorname{dim}(M)-3$.

Furthermore, if $N$ has negative sectional curvatures or if $\operatorname{dim} M=3$ and $N$ is any surface other than $S^{2}$ or $\mathbf{R P}^{2}$, then the map is completely regular. Since in these cases $N$ has a contractible covering space, the homotopy type of $g$ is determined by its 1-homotopy type. Consequently

THEOREM 4. If (1) $N$ has negative sectional curvatures, or

(2) $\operatorname{dim} M=3$ and $N$ is a surface other than $S^{2}$ or $\mathbf{R P}^{2}$, then every homotopy class of mappings from $M$ to $N$ contains a smooth map $g$ of least energy.

The main tools in the proofs are: a deformation procedure analogous to the Federer-Fleming one [F, 4.2.9], versions of the Poincaré and Sobolev inequalities that hold for polyhedral complexes (such as the $k$-skeleton of $M$ ), and the homotopy extension theorem. All of the results generalize in the expected way to manifolds $M$ with boundary.

Some special cases of these results were known previously: see [W2] and [W3] for details and references. Also, Theorem 4(1) was originally proved in a different way by Eells and Sampson [ES]. The analogous questions for 
area instead of energy are studied in [SU, SY] (when $\operatorname{dim} M=2$ ) and [W1] (when $\operatorname{dim} M>2$ ).

In [EL, II.2.4-5] it is pointed out that Theorem 4(2) follows from the case $p=2$ of Theorem 2. However, it seems that no proof (even in that case) has been published (though Schoen and Yau $[\mathbf{S Y}]$ gave a proof when $\operatorname{dim} M=p$ ).

I would like to thank $R$. Schoen for bringing these questions to my attention and for helpful conversations, and J. Eells for pointing out some errors.

\section{REFERENCES}

[EL] J. Eells and L. Lemaire, Selected topics in harmonic maps, CBMS Regional Conf. Ser. in Math., no. 50, Amer. Math. Soc., Providence, R. I., 1983.

[ES] J. Eells and J. Sampson, Harmonic mappings of Riemannian manifolds, Amer. J. Math. 86 (1964), 109-160. 1969.

[F] H. Federer, Geometric measure theory, Springer-Verlag, Berlin-Heidelberg-New York,

[SU] J. Sacks and K. Uhlenbeck, The existence of minimal 2-spheres, Ann. of Math. (2) 113 (1981), 1-24.

[SU1] R. Schoen and K. Uhlenbeck, $A$ regularity theory for harmonic maps, J. Differential Geom. 17 (1982), 307-335.

[SU2] , Boundary regularity and the Dirichlet problem for harmonic maps, $/$ J. Differential Geom. 18 (1983), 253-268.

[SY] R. Schoen and S. T. Yau, Existence of incompressible minimal surfaces and the topology of three-dimensional manifolds with non-negative scalar curvature, Ann. of Math. (2) 110 (1979), 127-142.

[W1] B. White, Mappings that minimize area in their homotopy classes, J. Differential Geom. (to appear).

[W2] _ Infima of energy functionals in homotopy classes of mappings, preprint.

[W3] _ Mappings that minimize energy functionals in their homotopy classes (in preparation).

DEPARTMENT OF MATHEMATICS, STANFORd UNIVERSity, StANFORD, CALIFORNIA 94305 